\title{
Association between sleep disordered breathing and aggressiveness markers of malignant cutaneous melanoma
}

\author{
Miguel-Ángel Martínez-García ${ }^{1,2}$, Antonio Martorell-Calatayud ${ }^{3}$, \\ Eduardo Nagore ${ }^{4,5}$, Irene Valero', Maria Jose Selma', Eusebi Chiner ${ }^{6}$, \\ Pedro Landete ${ }^{6}$, Josep-Maria Montserrat ${ }^{2,7}$, Cristina Carrera ${ }^{7}$, Amalia Pérez-Gil ${ }^{8}$, \\ Francisco Campos-Rodríguez ${ }^{8}$ and Ramón Farré2,9
}

Affiliations: 'Servicio de Neumología, Hospital Universitario y Politécnico La Fe, Valencia, ${ }^{2} \mathrm{CIBER}$ de Enfermedades Respiratorias, Bunyoles, ${ }^{3}$ Servicio de Dermatología, Hospital de Manises, Valencia, ${ }^{4}$ Instituto Valenciano de Oncología, Valencia, ${ }^{5}$ Facultad de Medicina, Universidad Católica de Valencia "San Vicente Martir", Valencia, 'Servicio de Neumología, Hospital Universitario San Juan, Alicante, "Servicios de Neumología y Dermatología, IDIBAPs Hospital Clinic, Barcelona, ${ }^{8}$ Servicios de Neumología y Dermatología, Hospital Valme, Seville, and 'Universidad de Barcelona-IDIBAPS, Barcelona, Spain.

Correspondence: M-Á. Martínez-García, Polytechnic and University La Fe, Hospital, Bulevar Sur s/n, 46026, Valencia, Spain. E-mail: mianmartinezgarciađgmail.com

ABSTRACT Some recent studies have shown an association between sleep disordered breathing (SDB) and cancer mortality and incidence but no study has focused on a specific type of cancer. The objective of this study was to analyse the relationship between the severity of SDB and factors related to cutaneous malignant melanoma (CMM) aggressiveness.

We performed a multicentre observational study in 82 consecutive patients diagnosed with CMM. 56 patients in whom melanoma measurements were available were finally included in the study. Melanoma measurements of aggressiveness included: tumour mitotic rate, Breslow index, presence of ulceration, stage of disease and growth rate of melanoma. A sleep study was performed in all the included patients. Multivariate analyses were used to examine the independent relationship between SDB severity (apnoeahypopnea index (AHI) and nocturnal oxygen desaturation indexes (ODI3\% and ODI4\%)) and measures of CMM aggressiveness.

$60.7 \%$ of patients had SDB (AHI $\geqslant 5)$ and $14.3 \%$ severe obstructive sleep apnoea (AHI $\geqslant 30)$. In fully adjusted multivariate analyses, AHI (OR 1.08, 95\% CI 1.02-1.14), ODI3\% (OR 1.08, 95\% CI 1.02-1.11) and ODI4\% (OR 1.1, 95\% CI 1.02-1.2) were independently associated with an increased melanoma growth rate. Furthermore, AHI, ODI $4 \%$ and ODI3\% were significantly correlated with other aggressiveness factors of CMM, such as Breslow index, presence of ulceration and mitotic index.

SDB severity markers are associated with some aggressiveness markers of CMM.

@ERSpublications

This study adds evidence on the relationship between sleep disordered breathing and cancer aggressiveness http://ow.ly/tMRYu

For editorial comments see page 1567.

Received: July 082013 | Accepted after revision: Feb 142014 | First published online: March 232014

Conflict of interest: None declared.

Copyright (OERS 2014 


\section{Introduction}

Sleep disordered breathing (SDB), and most particularly obstructive sleep apnoea (OSA), is characterised by repeated episodes of total or partial obstruction of the upper airways that trigger intermittent oxygen desaturation and disruption of the sleep architecture, leading to well-known consequences in the neurocognitive and cardiovascular domains [1].

Intermittent hypoxaemia is a typical process of SDB, with proven effects at the cellular level [2, 3]. Its presence has been associated with carcinogenesis and accelerated tumour growth [4]. However, the relationship between SDB and cancer has barely been studied, although there is some animal $[5,6]$ and clinical human evidence suggesting that an excess of SDB, particularly when related to intermittent hypoxaemia, is associated with an increase in the growth rate [5], incidence [7] and mortality [8, 9] of cancer. Nevertheless, none of the human studies has analysed any particular aetiology of cancer.

Cutaneous malignant melanoma (CMM) is a tumour of a melanocytic origin. Despite representing only $5 \%$ of all cutaneous tumours, it currently accounts for up to $75 \%$ of the deaths caused by cutaneous cancer. No effective treatment is available once this malignant tumour has spread, so the main efforts to control its annual incidence and associated mortality rate have focused on primary and secondary prevention [10-12]. In addition to some known prognostic values of CMM, such as the presence of ulceration, the Breslow index and the tumour mitotic rate (the number of mitotic cells per square millimetre), a simple calculation of the growth rate of melanoma (GRM) (in millimetres per month) has been published and validated by various authors, without any delay in tumour excision. A group of fast-growing melanomas has been identified, in which the tumour grows $\geqslant 0.5 \mathrm{~mm} \cdot \mathrm{month}^{-1}$. This group has been significantly correlated with several prognostic factors [13-17]. Their characterisation would be crucial to the application of more effective preventive measures. The main objective of the present study was to determine the relationship between the number and severity of SDB events and factors related to aggressiveness of CMM, including GRM.

\section{Material and methods}

Design

This was a multicentre, observational study performed in six centres (Hospital La Fe, Hospital de Manises and Instituto Valenciano de Oncología, Valencia; Hospital San Juan, Alicante; Hospital Clinic, Barcelona; Hospital Valme, Seville, Spain) with wide experience in the diagnosis and treatment of both SDB and CMM.

\section{Subjects}

The study included patients aged $>18$ years with invasive CMM diagnosed in the 12 months prior to their inclusion in the study, identified at the dermatology departments of each participating centre. Patients with daytime respiratory or heart failure, and those undergoing treatment with continuous positive airway pressure (CPAP), at-home oxygen therapy or noninvasive mechanical ventilation were excluded from the study. Patients with in situ melanomas and melanomas in an extracutaneous location were also excluded. All the patients gave their informed consent to participate, and all the centres' ethical committees approved the study protocol.

\section{Protocol and data collection}

\section{Dermatological protocol}

Information was collected by a senior dermatologist in each centre in accordance with a standardised protocol, including age, sex, body mass index (BMI) and location of the primary CMM (head or neck, upper extremities, trunk, lower extremities, or acral). The final diagnosis of CMM was based on a fullthickness excisional biopsy with a small side margin and made by an experienced pathologist in each centre. The histology report followed international guidelines and included information on the maximum thickness in millimetres (Breslow index), information on the tumour mitotic rate (number of mitotic cells per square millimetre) and the presence of ulceration. Finally, the stage of the disease was assessed according to the American Joint Committee on Cancer (7th edition). Localised melanoma was classified as stage IA, IB, IIA, IIB or IIC, locoregional disease as stage IIIA, IIIB or IIIC, or, finally, distant metastases as stage IV [18].

\section{Method for the evaluation of the growth rate of CMM}

Dermatologists calculated the GRM over time, as validated by various authors [13-17], on the basis of both information provided by patients and their families about changes in the skin lesion and the related histological findings. For these purposes, the date on which the patient noticed changes suggestive of malignant transformation in a stable, pre-existing lesion (such as increase in size, pigmentation, shape, symmetry, border, texture or diameter), or the time he/she noticed the appearance of a new and changing lesion was defined as D1. D2 was the date of the excision of the tumour, as recorded in the pathological report. The GRM was calculated as the Breslow index (in millimetres) divided by the difference between D2 
and D1 (in months). For the purposes of this study, the growth rate was categorised into two groups: slowintermediate growth rate $\left(<0.49 \mathrm{~mm} \cdot \mathrm{month}^{-1}\right)$ and fast growth rate $\left(\geqslant 0.5 \mathrm{~mm} \cdot \mathrm{month}^{-1}\right)$. Dermatological and pathological evaluations were blind with respect to the analysis of the sleep study.

\section{Sleep unit protocol}

The sleep visit involved the collection of data from the patient's clinical history with respect to sleep disorders (chronic snoring, witnessed apnoeas, Epworth Sleepiness Scale (ESS) score for daytime hypersomnia and anthropometric parameters such as BMI) at the time of the sleep study, as well as any characteristics that could act as confounding variables in the analysis of the results.

\section{Diagnostic sleep test}

All the patients underwent a diagnostic sleep test within a maximum period of 1 month after their visit to the sleep unit. In every case, the sleep test was carried out at home with respiratory polygraphy devices that had been appropriately validated in the literature. The following polygraphic or oximetric parameters were obtained: recording time, apnoea-hypopnoea index (AHI), baseline saturation, mean saturation, minimum saturation, night time with saturation below 90\% ( sat $_{90}$ ) and oxygen desaturation index at 3\% (ODI3\%) and $4 \%$ (ODI $4 \%)$. All the readings were carried out manually. Apnoea was defined as an interruption of oronasal airflow of $>10 \mathrm{~s}$. Hypopnoea was defined as a 30-90\% reduction in the oronasal airflow for $>10 \mathrm{~s}$ associated with an oxygen desaturation $\geqslant 4 \%$ or an arousal. AHI was defined as the number of apnoeas plus hypopneas per hour of sleep (polysomnography) or recording (respiratory polygraphy), while $T_{\text {sat }}{ }_{90}$ was defined as the percentage of night time with arterial oxygen saturation $<90 \%$. In all cases, oxygen saturation was measured at intervals of $2 \mathrm{~s}$ throughout the night. For a test to be considered valid, it had to involve both a subjective assessment by the patient of a minimum sleeping time of $5 \mathrm{~h}$ and a minimum recording time of $5 \mathrm{~h}$ of sufficient quality, after the elimination of spells of erratic recording. If these conditions were not met, the test was repeated. Any study in which any technical problems (particularly the disconnection of the pulse oximeter) were detected for $>10 \%$ of the total recording time was also repeated.

\section{Statistical analysis}

A statistical package (SPSS version 17; IBM, Armonk, NY, USA) was used for calculations. The quantitative variables are presented as mean $\pm \mathrm{SD}$ (or median (interquartile range (IQR)) in cases of non-normal distribution), while the qualitative or dichotomous variables are presented as $n(\%)$. The normality of the variables was confirmed by using the Kolmogorov-Smirnov test. The baseline variables of CMM with slowintermediate and fast growth rate were compared with the t-test or Mann-Whitney U-test, depending on whether the distribution was normal. Logistic regression analyses were performed to determine the independent association between respiratory parameters (AHI, ODI3\% and ODI4\%) and the two groups of GRM, using age, sex and BMI as confounders. Additionally, three linear regression analyses were performed, one for each respiratory parameter (AHI, ODI3\% and ODI4\%) as an independent variable using GRM as a quantitative dependent variable.

Finally, correlation analyses (Spearman test) were used to analyse the association between the polygraphic or oximetric parameters and the Breslow index, mitotic index, melanoma stage and growth rate of CMM as a continuous variable.

\section{Results}

Of 82 eligible patients, 56 were ultimately included in the analysis (fig. 1). There were no statistically significant differences in basal characteristics between the included and excluded patients (data not shown). The baseline characteristics of the studied patients and their CMM can be seen in table 1. Mean \pm SD age was $56.2 \pm 16.2$ years $(60.7 \%$ males $) .41$ patients had a local extension of their melanoma, 14 had a locoregional

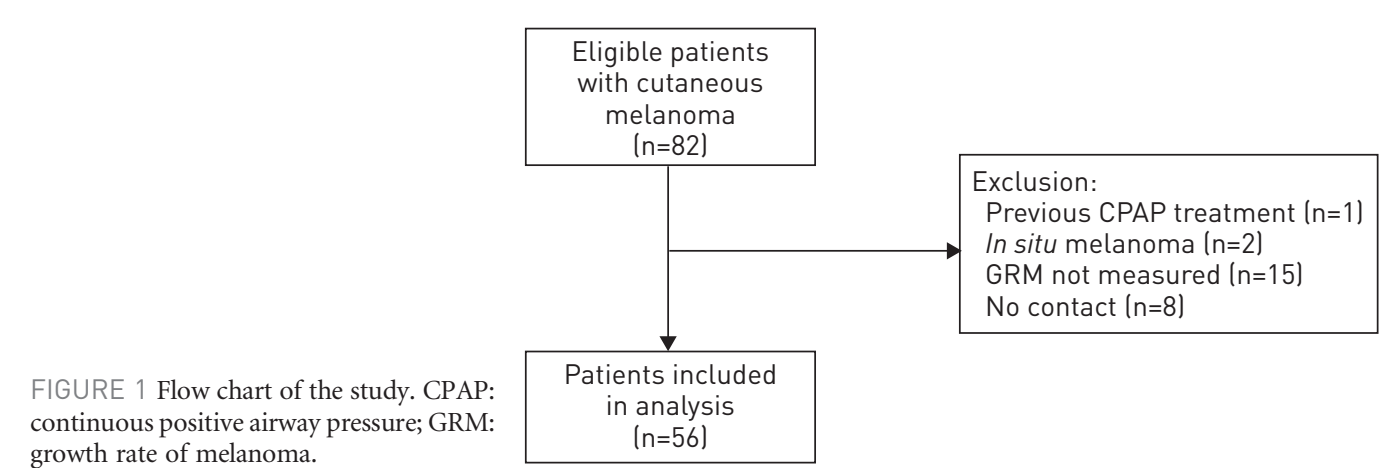




\section{TABLE 1 Baseline data of the patients, including melanoma characteristics}

\begin{tabular}{|c|c|}
\hline $\begin{array}{l}\text { Age years mean } \pm S D \\
\text { Males } \%\end{array}$ & $\begin{array}{c}56.2 \pm 16.2 \\
60.7\end{array}$ \\
\hline Breslow index mm & $2.26 \pm 2.4$ \\
\hline GRM mm.month ${ }^{-1}$ & $0.56 \pm 0.72$ \\
\hline Slow-intermediate GRM & $34(60.8)$ \\
\hline Fast GRM & 22 (39.2) \\
\hline Mitotic index cells $\cdot \mathrm{mm}^{-2}$ median (IQR) & $1(0-4)$ \\
\hline Ulceration & $14(25)$ \\
\hline \multicolumn{2}{|l|}{ Melanoma location } \\
\hline Head or neck & $7(12.5)$ \\
\hline Arms & $7(12.5)$ \\
\hline Legs & $11(19.6)$ \\
\hline Trunk & $28(50)$ \\
\hline Acral & $3(5.4)$ \\
\hline \multicolumn{2}{|l|}{ Disease stage } \\
\hline Localised melanoma" & $41(73.2)$ \\
\hline Locoregional disease & $14(25)$ \\
\hline Distant metastases $^{+}$ & $1(1.8)$ \\
\hline
\end{tabular}

Data are presented as mean \pm SD or $n(\%)$, unless otherwise stated. GRM: growth rate of melanoma; IQR: interquartile range. \# : stages IA (19 patients), IB (seven patients), IIA (nine patients), IIB (three patients) and IIC (three patients); ${ }^{\natural}$ : stages IIIA (four patients), IIIB (five patients) and IIIC (five patients); ${ }^{+}$: stage IV (one patient).

extension and one had metastasis. 34 melanomas had slow-intermediate GRM and 22 fast GRM. The time elapsed between CMM excision and the sleep study was $7.2 \pm 2.5$ months. No significant changes were observed in anthropometric data over this time. All the sleep studies were performed at home. The Breslow index was $2.27 \pm 2.4 \mathrm{~mm}$ and the median (IQR) mitotic index was $1(0-4)$ cells $\cdot \mathrm{mm}^{-2}$.

\section{Prevalence of SDB}

Table 2 shows the prevalence of SDB in our series of patients with CMM. The mean AHI was $13.6 \pm 17.3$, with an ODI3\% of $14.4 \pm 17.9$ and an ODI $4 \%$ of $8.28 \pm 13.5$. The mean ESS score was $6.8 \pm 4.14 .60 .7 \%$ of patients presented with an $\mathrm{AHI} \geqslant 5,35.7 \%$ an $\mathrm{AHI} \geqslant 10,30.3 \%$ an $\mathrm{AHI} \geqslant 15$ and $14.3 \%$ an $\mathrm{AHI} \geqslant 30$.

\section{Relationship between SDB and factors of melanoma aggressiveness}

Table 3 shows the clinical, pathological and polygraphic results of the two groups of patients according to their GRM. It is noteworthy that there were significant differences in both the AHI and intermittent hypoxaemia variables (ODI3\% and ODI4\%) between groups. However, no significant differences were found between groups in age, sex, BMI or daytime hypersomnia.

\section{TABLE 2 Results from the sleep studies}

$\begin{array}{lc}\text { Chronic snore } & 30(53.6) \\ \text { Witnessed apnoeas } & 9(16.1) \\ \text { ESS score } & 6.8 \pm 4.14 \\ \text { BMI } \mathbf{~ g g} \cdot \mathbf{m}^{-2} & 26.8 \pm 4.19 \\ \text { AHI events per } \mathbf{h} & 13.6 \pm 17.3 \\ A H I \geqslant 5 & 34(60.7) \\ A H I \geqslant 10 & 20(35.7) \\ A H I \geqslant 15 & 17(30.3) \\ A H I \geqslant 30 & 8(14.3) \\ \text { Basal saturation \% } & 96.3 \pm 1.9 \\ \text { Mean saturation \% } & 94.1 \pm 2.5 \\ \text { ODI3\% events per } \mathbf{h} & 14.4 \pm 17.9 \\ \text { ODI4\% events per } \mathbf{h} & 8.28 \pm 13.5 \\ \text { Tsat }_{90} \% & 8.2 \pm 19.6 \\ \text { Minimum saturation \% } & 83.9 \pm 8.1\end{array}$

Date are presented as $\mathrm{n}(\%)$ or mean \pm SD. ESS: Epworth Sleepiness Scale; BMI: body mass index; AHI: apnoeahypopnea index; ODl3\%: oxygen desaturation index at 3\%; 0DI4\%: oxygen desaturation index at 4\%; sat $_{90}$ : night time spent with oxygen saturation $<90 \%$. 


\begin{tabular}{|c|c|c|c|}
\hline \multirow[t]{2}{*}{ Variable } & \multicolumn{2}{|c|}{ GRM } & \multirow[t]{2}{*}{ p-value } \\
\hline & Slow-intermediate & Fast & \\
\hline Subjects $n$ & 34 & 22 & \\
\hline Age years & $52.9 \pm 14.4$ & $61.3 \pm 17.8$ & 0.06 \\
\hline Males & $20(58.8)$ & $14(63.6)$ & 0.72 \\
\hline Ulceration & 6.06 & $12(54.4)$ & 0.0001 \\
\hline Mitotic index cells $\cdot \mathrm{mm}^{-2}$ & $0.8 \pm 1.7$ & $5.36 \pm 1.19$ & 0.0001 \\
\hline Mitotic index $\geqslant 5$ & $2(\overline{6} .4)$ & $8(36.4)$ & 0.008 \\
\hline Breslow index mm & $1.03 \pm 0.88$ & $4.17 \pm 2.73$ & 0.0001 \\
\hline GRM mm $\cdot$ month $^{-1}$ & $0.15 \pm 0.12$ & $1.19 \pm 0.79$ & 0.0001 \\
\hline \multicolumn{4}{|l|}{ Melanoma localisation } \\
\hline Neck/head/acral/trunk & $23(67.6)$ & 15 (68.2) & 0.91 \\
\hline Legs/arms & $11(32.6)$ & 7 (31.8) & 0.96 \\
\hline ESS score & $7.42 \pm 4.6$ & $5.8 \pm 3.1$ & 0.07 \\
\hline AHI events per $h$ & $6.3 \pm 6$ & $25 \pm 22.4$ & 0.0001 \\
\hline$A H I \geqslant 5$ & $16(47.1)$ & $17(77.3)$ & 0.04 \\
\hline$A H I \geqslant 10$ & $6(17.7)$ & $14(63.6)$ & 0.0001 \\
\hline$A H I \geqslant 30$ & $1(3)$ & 7 (31.8) & 0.002 \\
\hline $\mathrm{BMI} \mathbf{k g} \cdot \mathrm{m}^{-2}$ & $26.3 \pm 4.2$ & $27.7 \pm 4.2$ & 0.26 \\
\hline Basal saturation \% & $96.9 \pm 1.54$ & $95.2 \pm 2.1$ & 0.016 \\
\hline Mean saturation \% & $94.5 \pm 2$ & $93.5 \pm 3.1$ & 0.15 \\
\hline ODI3\% events per $\mathrm{h}$ & $6.4 \pm 5.4$ & $26.6 \pm 22.9$ & 0.0001 \\
\hline ODIL\% events per $\mathrm{h}$ & $2.38 \pm 2.81$ & $17.1 \pm 17.9$ & 0.0001 \\
\hline$T_{\text {sat }_{90}} \%$ & $2.3 \pm 6.1$ & $17.2 \pm 28.3$ & 0.005 \\
\hline Minimum saturation \% & $86.4 \pm 5.3$ & $80.1 \pm 10.3$ & 0.004 \\
\hline
\end{tabular}

Data are presented as mean \pm SD or $n(\%)$, unless otherwise stated. All $p<0.05$ between fast and slowintermediate GRM, except for age. ESS: Epworth Sleepiness Scale; AHI: apnoea-hypopnea index; BMI: body mass index; ODI3\%: oxygen desaturation index at 3\%; ODI4\%: oxygen desaturation index at 4\%; sat $_{90}$ : night time spent with oxygen saturation $<90 \%$.

Tables 4 and 5 show the multivariate analyses performed to assess the independent relationship between various respiratory parameters in the sleep study (AHI, ODI3\% and ODI4\%) and the GRM. In table 4, a logistic regression analysis was used considering GRM the dependent variable, as a dichotomous variable (fast versus slow-intermediate GRM), and in table 5, a linear multivariate analysis was performed considering GRM as a continuous variable. All models were adjusted for age, sex and BMI. Both multivariate approaches show that AHI, ODI3\% and ODI4\% were independently associated with GRM considered as both quantitative and dichotomous variables.

Figure 2 shows a positive correlation between the Breslow index and AHI $(\mathrm{r}=0.37, \mathrm{p}=0.005)$, ODI $3 \%$ $(\mathrm{r}=0.36, \mathrm{p}=0.005)$ and ODI $4 \%(\mathrm{r}=0.38, \mathrm{p}=0.005)$. There were also positive correlations between the GRM and the AHI $(r=0.37, p=0.005)$, ODI $3 \%(r=0.43, p=0.001)$ and ODI $4 \%(r=0.43, p=0.001)$, and between the mitotic index and ODI3\% without reaching statistical significance $(r=0.24, \mathrm{p}=0.07)$. In patients with

TABLE 4 Relationship between sleep disordered breathing parameters and growth rate of melanoma as a dichotomous variable

Unadjusted OR $(95 \% \mathrm{Cl})$

Fully adjusted OR $(95 \% \mathrm{Cl})$

\begin{tabular}{llc}
\hline Model 1 & $1.09(1.03-1.15)^{\#}$ & $1.08(1.02-1.14)^{+}$ \\
Model 2 & $1.09(1.03-1.15)^{\#}$ & $1.08(1.02-1.11)^{\S}$ \\
Model 3 & $1.22(1.03-1.23)^{\oplus}$ & $1.1(1.02-1.2)^{f}$
\end{tabular}

Logistic regression models using apnoea-hypopnoea index as a dependent variable in model 1, oxygen desaturation index (ODI) $3 \%$ in model 2 and $\mathrm{ODI} 4 \%$ in model 3 . The unadjusted models only included polygraphic or oximetric parameters. The full adjusted models also included age, sex and body mass index. ${ }^{\#}: p=0.003$; †: $p=0.012 ;{ }^{+}: p=0.006 ;{ }^{\S}: p=0.008 ;{ }^{f}: p=0.023$. 
TABLE 5 Relationship between sleep disordered breathing parameters and growth rate of melanoma as a continuous variable

\begin{tabular}{lcccc} 
& $\boldsymbol{\beta}$-coefficient & Student's t & p-value & 95\% Cl \\
\hline Model 1\# & 0.033 & 4.16 & 0.001 & $0.017-0.049$ \\
Model 2 $^{*}$ & 0.028 & 0.46 & 0.004 & $0.009-0.047$ \\
Model 3+ $^{+}$ & 0.026 & 3.26 & 0.002 & $0.010-0.042$ \\
\hline
\end{tabular}

Logistic regression models using apnoea-hypopnoea index as a dependent variable in model 1, oxygen desaturation index (ODI) $3 \%$ in model 2 and ODI $4 \%$ in model 3 , and age, sex and body mass index as confounders. ${ }^{\#}: \mathrm{R}^{2}=0.29 ;{ }^{\bullet}: \mathrm{R}^{2}=0.21 ;{ }^{+}: \mathrm{R}^{2}=0.22$.

severe OSA (AHI $\geqslant 30)$, the mitotic index was significantly higher in comparison with the remaining patients $(5.37 \pm 7.56$ versus $1.83 \pm 2.9, \mathrm{p}=0.023)$. Furthermore, patients with an AHI $\geqslant 30(\mathrm{p}=0.06)$, ODI3\% $\geqslant 10(\mathrm{p}=0.08)$ or ODI $4 \% \geqslant 10(\mathrm{p}=0.08)$ showed a trend towards tumour ulceration.

Finally, considering the nine stages of melanoma as a ordinal variable (from IA to IV), there was a positive statistically significant correlation between the stage of melanoma at the moment of the excision and AHI $(\mathrm{r}=0.23, \mathrm{p}=0.046)$, ODI $3 \%(\mathrm{r}=0.28, \mathrm{p}=0.036)$ and ODI $4 \%(\mathrm{r}=0.25, \mathrm{p}=0.041)$.

\section{Discussion}

This is the first study in the literature to analyse the relationship between the presence and severity of SDB and their oximetric consequences, and prognostic variables of a specific type of cancer, in this case CMM. In our series, we found a positive and independent association between the AHI and ODI, as markers of SDB severity, and the GRM, as well as a positive association with other markers of aggressiveness of CMM, such as the Breslow index, the presence of ulceration, the mitotic index and the melanoma stage.

There are various pathophysiological mechanisms that link intermittent hypoxaemia and the formation or spread of cancer, particularly via key intermediate molecules in this process, such as hypoxia-inducible factor-1, a carcinogenesis-related molecule, and vascular endothelial growth factor, which promotes neovascularisation of the tumour and, therefore, its expansion [2, 3, 19]. According to this hypothesis, several studies in both animals and humans have shown that the presence of intermittent hypoxaemia (sometimes related to sleep apnoea) is associated with increased tumour growth [5], or higher cancer incidence [7] and mortality [8,9], in both population and clinical series. However, no studies to date have analysed the effects in patients diagnosed with a particular type of cancer (in this case CMM, as the growth rate of this tumour has proved sensitive to the presence of intermittent hypoxaemia, particularly in animal studies) [5].

One of the major advantages of studying factors related to the natural history of CMM is the fact that these tumours are, for the most part, visible and, therefore, any changes indicative of a malignant transformation can be identified by the patients or their relatives with some degree of trustworthiness. These changes on the surface of the tumour have shown a good correlation with its invasiveness and depth [13-17]. This
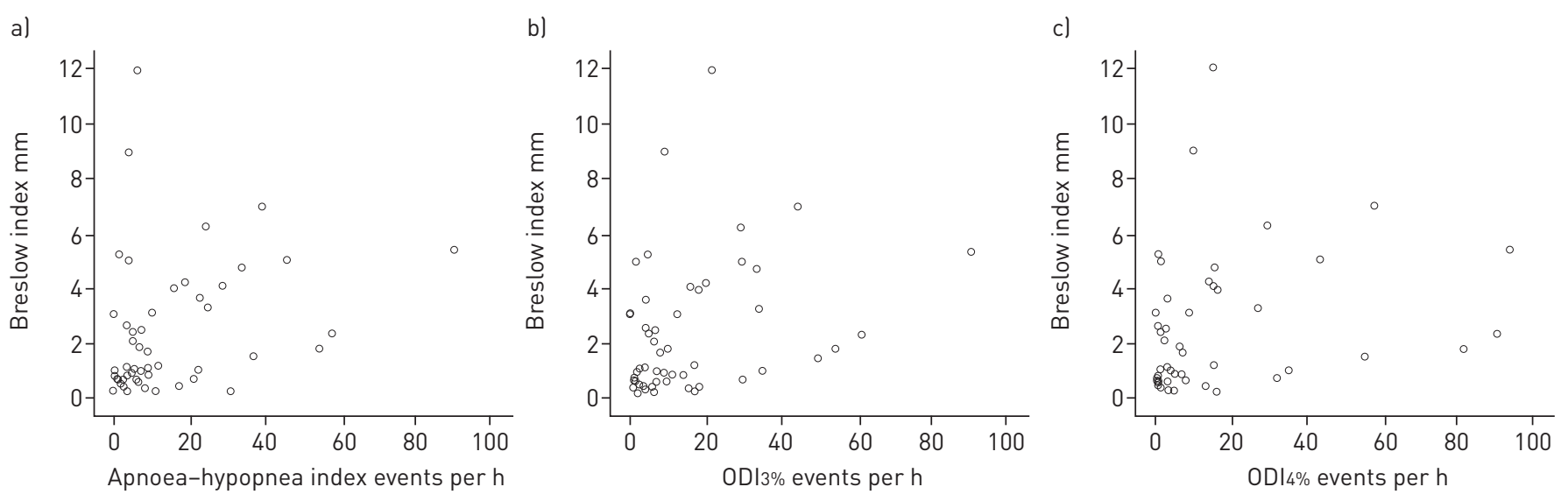

FIGURE 2 Correlation between Breslow index and a) apnoea-hypopnoea index, b) oxygen desaturation index at 3\% (ODI3\%) and c) oxygen desaturation index at $4 \%$ (ODI $4 \%)$. 
information, along with the Breslow index (thickness of the tumour measured in millimetres), allowed us to calculate the growth rate of CMM without delaying excision and to divide the CMMs into slow or fast GRM [13-17]. This classification has been significantly correlated with several prognostic factors of vital importance to the prognosis of CMM, such as the presence of metastasis, the thickness of the CMM, survival, the presence of ulceration, the mitotic index and the metastasis in the sentinel lymph node [13-17].

The first striking finding extracted from our results is that the prevalence of SDB in our patients with CMM was higher than that expected in the general population, when compared with epidemiological studies in patients of similar age and sex [20,21], even though the patients only presented a slight increase in BMI (mean BMI $26.8 \mathrm{~kg} \cdot \mathrm{m}^{-2}$ ) without excessive diurnal hypersomnia measured by the ESS (a key marker of SDB clinical severity).

The main result of our study, however, is that the presence of SDB and its oximetric consequences is significantly and independently associated with the growth rate of CMM, which seems to be independent of other risk factors for CMM and its aggressiveness, such as age and sex. Moreover, we observed a positive correlation of those polygraphic and oximetric parameters with other markers of aggressiveness, such as the presence of ulceration, the mitotic rate and the Breslow index. This serves to support the hypothesis that intermittent hypoxaemia caused by excessive SDB may promote faster growth or aggressiveness in patients suffering from CMM, although the methodological limitations of our study make it necessary to confirm our results in larger studies.

While the main strength of this study is that it is the first in the literature to investigate the relationship between SDB and its oximetric consequences, and parameters indicating the aggressiveness of a certain type of cancer, in this case CMM, it also presents several limitations. Probably the most relevant confounder is age, as it is an important factor in determining whether any newly diagnosed melanoma will display aggressive growth rate due to greater exposure to etiological factors [22] and age is associated with increased SDB [23]. In this sense, we observed a nearly significant difference between the two groups of GRM $(\mathrm{p}=0.06$, with a clinically significant difference of 8.4 years). To minimise the impact of age in our study, we included it as an adjusted variable in all the multivariate analyses performed. On the one hand, this is a small study, albeit one that has already brought to light some significant associations, while needing stronger statistical power (a greater number of patients) to prove others. Moreover, GRM measurement depends on patients' or relatives' recall and, therefore, it should be considered a subjective characteristic. On the other hand, it should be pointed out that it has been consistently demonstrated to be an independent prognostic factor for overall and disease-free survival [13-17]. Finally, this study has a retrospective nature regarding the diagnosis of $\mathrm{CMM}$, as the patients had been diagnosed with this tumour in the year prior to inclusion in the study. It must be stressed, however, that between the diagnosis of CMM and its excision, and the performance of the sleep test, we did not identify any significant changes in weight or in the presence of new confounding variables.

In conclusion, our study shows that the frequency and severity of SDB were independently associated with a higher GRM, and correlated with greater tumour thickness, a higher mitotic index and more frequent ulceration, all features associated with worse prognosis in CMM. Larger, prospective studies in patients with de novo CMM diagnoses are needed to confirm our conclusions, as these results, if confirmed, would offer an important clinical applicability in these patients, as well as a gateway to new therapeutic possibilities for this excess of SDB with CPAP in patients with a tumour as highly aggressive as CMM.

\section{References}

1 Lloberes P, Durán-Cantolla J, Martínez-García MA, et al. Diagnosis and treatment of sleep apnea-hypopnea syndrome. Spanish Society of Pulmonology and Thoracic Surgery. Arch Bronconeumol 2011; 47: 143-156.

2 Levy P, Pépin JL, Arnaud C, et al. Intermittent hypoxia and sleep-disordered breathing: current concepts and perspectives. Eur Respir J 2008; 32: 1082-1095.

Semenza GL. Oxygen sensing, homeostasis and disease. New Engl J Med 2011; 365: 537-547.

4 Toffoli S, Michiels C. Intermittent hypoxia is a key regulator of cancer cell and endothelial cell interplay in tumours. FEBS J 2008; 275: 2991-3002.

5 Almendros I, Montserrat JM, Ramírez J, et al. Intermittent hypoxia enhances cancer progression in a mouse model of sleep apnoea. Eur Respir J 2012; 39: 215-216.

6 Almendros I, Montserrat JM, Torres M, et al. Intermittent hypoxia increase melanoma metastasis to the lung in a mouse model of sleep apnea. Respir Physiol Neurobiol 2013; 186: 303-307.

7 Campos-Rodriguez F, Martínez-García MA, Martínez M, et al. Association between obstructive sleep apnea and cancer incidence in a large multicenter Spanish cohort. Am J Respir Crit Care Med 2013; 187: 99-106.

8 Nieto FJ, Peppard P, Young T, et al. Sleep disordered breathing and cancer mortality: results from the Wisconsin Sleep Cohort Study. Am J Respir Crit Care Med 2012; 186: 190-194.

9 Martínez-García MA, Campos-Rodríguez F, Duran-Cantolla J, et al. Association between nocturnal hypoxemia and cancer mortality. Longitudinal multicenter study in 5,467 patients from the Spanish Cohort. Eur Respir J 2012; 40: Suppl. 56, 709s.

10 Gorbe C, Leiter U. Melanoma epidemiology and trends. Clin Dermatol 2009; 27: 3-9. 
11 Tsao H, Atkins MB, Sober AJ. Management of cutaneous melanoma. N Engl J Med 2004; 35: 998-1012.

12 Thompson JF, Scolyer RA, Kefford RF. Cutaneous melanoma. Lancet 2005; 365: 687-701.

13 Tejera-Vaquerizo A, Nagore E, Herrera-Acosta E, et al. Prediction of sentinel lymph node positivity by growth rate of cutaneous melanoma. Arch Dermatol 2012; 148: 577-584.

14 Tejera-Vaquerizo A, Nagore E, Meléndez JJ, et al. Chronology of metastasis in cutaneous melanoma: Growth rate model. J Invest Dermatol 2012; 132: 1215-1221.

15 Grob JJ, Richard MA, Gouvernet J, et al. The kinetics of the visible growth of a primary melanoma reflects tumor aggressiveness and is an independent prognostic marker: a prospective study. Int J Cancer 2002; 102: 34-38.

16 Martorell-Calatayud A, Nagore E, Botella-Estrada R, et al. Defining fast-growing melanomas: reappraisal of epidemiological, clinical, and histological features. Melanoma Res 2011; 21: 131-138.

17 Nagore E, Martorell-Calatayud A, Botella-Estrada P, et al. Growth rate as an independent prognostic factor in localized invasive cutaneous melanoma. J Eur Acad Dermatol Venereol 2011; 25: 618-620.

18 Balch CM, Gershenwald JE, Soong SJ, et al. The American Joint Committee on Cancer (AJCC) classification. Final version of 2009 AJCC melanoma staging and classification. J Clin Oncol 2009; 27: 6199-6206.

19 Martínez-García MA, Campos-Rodriguez F, Farré R. Sleep apnea and cancer: current insights and future perspectives. Eur Respir 2012; J, 40: 1315-1317.

20 Young T, Palta M, Dempsey J, et al. The occurrence of sleep disordered breathing among middle age adults. N Engl J Med 1993; 328: 1230-1236.

21 Duran J, Esnaola S, Rubio R, et al. Obstructive sleep apnea-hypopnea and related clinical features in a populationbased sample of subjects aged 30 to 70 years. Am J Respir Crit Care Med 2001; 163: 685-689.

22 Gandini S, Sera F, Cattaruzza MS, et al. Meta-analysis of risk factors for cutaneous melanoma. III. Family history, actinic damage and phenotypic factors. Eur J Cancer 2005; 41: 2040-2059.

23 Bixler EO, Vgontzas AN, Ten Have T, et al. Effects of age on sleep apnea in men. Prevalence and severity. Am Respir J Crit Care Med 1998; 157: 144-148. 